\title{
Estimating dispersal potential for marine larvae: dynamic models applied to scleractinian corals
}

\author{
SeAn R. Connolly ${ }^{1,2,3}$ and Andrew H. Baird ${ }^{1}$ \\ ${ }^{1}$ ARC Centre of Excellence for Coral Reef Studies, James Cook University, Townsville, Queensland 4811 Australia \\ ${ }^{2}$ School of Marine and Tropical Biology, James Cook University, Townsville, Queensland 4811 Australia
}

\begin{abstract}
Dispersal influences ecological dynamics, evolution, biogeography, and biodiversity conservation, but models of larval dispersal in marine organisms make simplifying assumptions that are likely to approximate poorly the temporal dynamics of larval survival and capacity for settlement. In particular, larval mortality rates are typically assumed to be constant throughout larval life; and all larvae are frequently assumed to acquire and lose competence at the same time. To improve upon these assumptions, we here develop simple models of dispersal potential that incorporate rates of mortality, and acquisition and loss of settlement competence. We fit these models to empirical competence and survival data for five scleractinian coral species, to test the models' ability to characterize empirical survival and competence patterns, and to estimate the dispersal potential implied by those patterns. The models fit the data well, incorporating qualitative features of competence and survival that traditional approaches to modeling dispersal do not, with important implications for dispersal potential. Most notably, there was high within-cohort variation in the duration of the competent period in all species, and this variation increases both self-recruitment and longdistance dispersal compared with models assuming a fixed competent period. These findings help to explain the seeming paradox of high genetic population structure, coupled with large geographic range size, observed in many coral species. More broadly, our approach offers a way to parsimoniously account for variation in competence dynamics in dispersal models, a phenomenon that our results suggest has important effects on patterns of connectivity in marine metapopulations.
\end{abstract}

Key words: connectivity; coral reefs; dispersal model; larval settlement; larval survival.

\section{INTRODUCTION}

Dispersal plays a key role in the dynamics of populations and communities. It can influence the degree of spatial synchrony in population dynamics (Bjornstad et al. 1999), the coexistence and relative abundances of species (Mouquet and Loreau 2003), the potential for localized genetic drift and adaptation in populations (Bohonak 1999), and the geographical distribution of species (Lester et al. 2007). Consequently, an understanding of dispersal is important for answering a broad range of ecological and evolutionary questions, as well as for anticipating the effects of habitat fragmentation (Tilman et al. 1997), and for designing networks of protected areas (Almany et al. 2009).

Many organisms, including plants, arthropods, and marine fishes and invertebrates, have a relatively sedentary adult phase and a dispersive phase, during which physical processes like winds and ocean currents can transport propagules great distances (Nathan and Muller-Landau 2000, Compton 2002, Strathmann et al. 2002). In the marine realm, the predominant view for

Manuscript received 20 January 2010; revised 10 May 2010; accepted 11 May 2010. Corresponding Editor: S. G. Morgan.

${ }^{3}$ E-mail: sean.connolly@jcu.edu.au much of the last half-century was that larvae of benthic organisms are dispersed widely, and therefore that subpopulations are demographically well connected over large spatial scales, relative to terrestrial organisms (Palumbi 1994). This is consistent with the large geographic ranges of many marine taxa (Hughes et al. 2002, Paulay and Meyer 2002), and with comparative estimates of dispersal distance from genetic data (Kinlan and Gaines 2003). However, recent work also suggests that a large proportion of marine larvae may be retained locally, even in species with extended larval durations (Sponaugle et al. 2002, Kinlan and Gaines 2003, Hellberg 2007).

Attempts to understand the ecological and evolutionary effects of dispersal have included the development of models that incorporate physical and biological determinants of successful dispersal (Cowen and Sponaugle 2009). The characterization of physical processes in such models has become highly sophisticated (Cowen et al. 2006, Siegel et al. 2008). In contrast, the characterization of larval biology in most dispersal models tends to use highly idealized assumptions; for instance, that larval mortality is constant, and that all larvae in a cohort acquire and/or lose competence simultaneously (e.g., Cowen et al. 2006, Siegel et al. 2008), although both assumptions are likely to be violated in nature (Strath- 
mann 1974, Rumrill 1990, Graham et al. 2008). At the other extreme, a few models infer survival and settlement from individual-based sub-models of foraging and growth (e.g., Vikebo et al. 2007). However, because such models tend to be very parameter rich, there are rarely sufficient data to calibrate them (Gallego et al. 2007).

The dispersal of broadcast-spawning scleractinian corals is likely to be particularly sensitive to assumptions about larval survival and the onset and duration of settlement competence. Coral larvae become competent to settle within a few days (Baird 2001, Miller and Mundy 2003, Nozawa and Harrison 2008). Retention times of water masses on coral reefs are of similar duration (Black et al. 1991), suggesting that the extent of local self-recruitment may be particularly sensitive to small variations in larval development times or local circulation characteristics. The swimming speeds of coral larvae are substantially lower than most other marine invertebrate larvae (Chia et al. 1984), and thus less effective at reducing sensitivity to physical transport mechanisms than many other taxa. At the other end of the larval phase, little is known about the maximum time that larvae retain competence, because most studies expose larvae to settlement cues continuously, or terminate before settlement ceases (e.g., Ben-DavidZaslow and Benayahu 1998, Nozawa and Harrison 2008). Moreover, recent work suggests that mortality rates are elevated both early and late in larval lifetimes (Graham et al. 2008), in violation of the assumption of constant mortality characteristic of most models of larval dispersal. This has implications for the proportion of larvae surviving long enough to be transported off the natal reef, and subsequently for the potential to remain alive for very long-distance dispersal. Indeed, the prevalence of local retention vs. long-distance dispersal is particularly enigmatic for corals, given that they tend to have extremely large ranges (e.g., a median longitudinal extent of 109 degrees; Hughes et al. 2002), but also local genetic structure and stock-recruitment relationships indicative of substantial local retention (Hughes et al. 2000, van Oppen and Gates 2006). Historically, this led to the invocation of unique mechanisms for long versus short-distance dispersal, such as rafting of coral recruits on floating pumice (Jokiel 1990).

In this study, we formulate, empirically calibrate, and analyze models for the dynamics of survival and settlement competence in scleractinian corals. Our models characterize such dynamics more realistically than most dispersal models, but are parsimonious enough to be calibrated with readily obtainable empirical data. Therefore, we collect data on survival and settlement competence for five species of scleractinian corals, and we use these data to calibrate our models. Finally, we use our results to estimate dispersal potential in the study species, and we compare those estimates with estimates based on models that make the more traditional assumptions of constant mortality rates, and no within-cohort variation in competence periods. These comparisons reveal surprising effects of within-cohort variation in competence on dispersal potential that may help to resolve the seeming paradox of large geographic ranges and fine-scale population structure that is characteristic of scleractinian corals. These models also offer a framework for characterizing more realistically, but parsimoniously, the dynamics of survival and competence in coupled biophysical dispersal models, a characterization that our results indicate has important effects on patterns of connectivity in marine metapopulations.

\section{A Model of Dispersal Potential}

We conceptualize the larval phase of scleractinian corals as consisting of up to three phases: precompetent, competent, and post-competent. During the pre-competent phase, development is still underway, and larvae are not yet competent to settle. During the competent phase, larvae are capable of settlement. Postcompetent larvae have lost the capacity to successfully settle, but they remain alive (Richmond 1987, BenDavid-Zaslow and Benayahu 1998). Specifically, we model the dynamics of a larval cohort as follows:

$$
\begin{aligned}
& \frac{d L_{1}}{d t}=-\alpha(t) L_{1}(t)-\mu(t) L_{1}(t) \\
& \frac{d L_{2}}{d t}=\alpha(t) L_{1}(t)-\beta(t) L_{2}(t)-\mu(t) L_{2}(t) \\
& \frac{d L_{3}}{d t}=\beta(t) L_{2}(t)-\mu(t) L_{3}(t)
\end{aligned}
$$

with initial conditions

$$
L_{1}(0)=1 \quad L_{2}(0)=0 \quad L_{3}(0)=0 .
$$

The subscripts 1, 2, and 3 refer to the pre-competent, competent, and post-competent larval stages, respectively; $L_{i}(t)$ is the proportion of larvae that are alive and in stage $i$ at time $t ; \mu(t)$ is the per-capita mortality rate at time $t ; \alpha(t)$ is the per-capita rate of acquisition of settlement competence at time $t ; \beta(t)$ is the corresponding rate of loss of competence.

To keep the number of model parameters to a minimum, we begin with the simplifying assumptions that the acquisition and loss of competence occur at constant per-capita rates: $\alpha(t)=a, \beta(t)=b$. We then solve the system of Eqs. 1 and 2 explicitly to obtain the proportion of the initial cohort of larvae that is alive and competent as a function of time:

$$
L_{2}(t)=\frac{a\left(e^{-a t}-e^{-b t}\right)}{b-a} s(t)
$$

where $s(t)$ is the proportion of the initial cohort that is still alive at time $t$,

$$
s(t)=\exp \left[-\int_{z=0}^{t} \mu(z) d z\right] .
$$


To obtain the proportion of surviving larvae that are competent, we normalize Eq. 3 by the total proportion of the initial cohort that is still alive at time $t$ :

$$
P_{2}(t)=\frac{L_{2}(t)}{L_{1}(t)+L_{2}(t)+L_{3}(t)}=\frac{a\left(e^{-b t}-e^{-a t}\right)}{a-b} .
$$

Note that Eq. 5 is independent of the mortality rate, $\mu(t)$, so mortality parameters can be estimated separately from the competence parameters, $a$ and $b$ (see Empirical methods).

Eq. 1 assumes that acquisition of competence begins immediately. However, species that broadcast-spawn gametes, including $85 \%$ of all scleractinians (and all species used in this study), have an obligate planktonic period of $\sim 12-36 \mathrm{~h}$, during which embryogenesis occurs and larvae become motile (Harrison and Wallace 1990, Baird et al. 2009). Additional time may be required to develop the capacity to attach to the substrate and metamorphose (Paruntu et al. 2000). One way to approximate such a time delay parsimoniously is to assume that the rate of acquisition of competence is zero until this obligate period has expired:

$$
\alpha(t)= \begin{cases}0 & t<t_{\mathrm{c}} \\ a & t>t_{\mathrm{c}}\end{cases}
$$

where $t_{\mathrm{c}}$ is the development time required before acquisition of competence can begin. With this modification, the proportion of surviving larvae that are competent at time $t$ is

$$
P_{2}^{*}(t)= \begin{cases}0 & t<t_{\mathrm{c}} \\ \frac{a\left(e^{-b\left(t-t_{\mathrm{c}}\right)}-e^{-a\left(t-t_{\mathrm{c}}\right)}\right)}{a-b} & t>t_{\mathrm{c}}\end{cases}
$$

where the asterisk indicates the time-delay model.

For mortality, we considered both constant and timevarying rates. If mortality rate is constant, the proportion of an initial cohort that is still alive exhibits an exponential decline

$$
s(t)=e^{-\lambda t}
$$

where $\lambda$ is the instantaneous mortality rate. To allow for monotonically increasing or decreasing mortality rates, we use a Weibull distribution, whose corresponding expression is

$$
s(t)=\exp \left[-(\lambda t)^{v}\right]
$$

where $\lambda>0, v>0$. If $v>1$, then mortality rate increases with age; if $v<1$, it decreases with age. When $v=1$, Eq. 9 collapses to the exponential model (Eq. 8). A third possibility is that mortality rates are high initially, decrease, and then increase again late in larval life. To allow for this possibility, we fit a "generalized Weibull" distribution. For this model, the proportion of a cohort still alive at time $t$ is

$$
s(t)=\left(1-\sigma[\lambda t]^{v}\right)^{1 / \sigma}
$$

where $\lambda>0, v>0, \sigma<\left(\lambda t_{\max }\right)^{-v}$, and $t_{\max }$ is the last day on which larval abundance was estimated (Mudholkar et al. 1996). Depending on the values of the shape parameters $\sigma$ and $v$, mortality rate may be increasing, decreasing, "bathtub-shaped" (decreasing, then increasing), or hump-shaped. In the limit as $\sigma \rightarrow 0$, this model is equivalent to Eq. 9.

Eq. 3 is temporal dispersal potential: the proportion of the cohort that is alive and competent as a function of time. Such a model can be combined with physical models of larval transport, and of the spatial distribution of reefs, to predict larval dispersal. Because both transport processes and the spatial distributions of habitat patches are highly context-specific, this will lead to different dispersal kernels in different reef systems and at different times. Nevertheless, to illustrate how temporal dispersal potential can be translated into spatial dispersal potential, we apply a simple generic model of physical transport, in which diffusion produces a Gaussian distribution whose width depends on the strength of diffusive processes, $k$, and time spent being transported, $t$ :

$$
D(x, t)=\frac{1}{\sqrt{4 \pi k t}} e^{\left(-x^{2}\right) /(4 k t)} .
$$

Advection shifts this distribution downstream, but does not change its shape (Largier 2003). Combining Eq. 3 and Eq. 11, we obtain dispersal potential as a function of distance:

$$
L_{2}(x)=\int_{t=t_{\mathrm{c}}}^{\infty} D(x, t) L_{2}(t) d t
$$

$\left(t_{\mathrm{c}}=0\right.$ for the model without a time lag).

Eq. 12 provides a useful basis for assessing differences between species in, and effects of particular model assumptions on, the potential for short and long-distance dispersal. Note, however, that it does not assume a particular distribution of habitat patches, and thus does not predict where propagules will ultimately settle. Rather, it gives the proportion of larvae that will reach location $x$ while alive and competent to settle (i.e., the proportion of larvae that would be able to settle at $x$ ) if they had not had an opportunity to settle previously. It is therefore a spatial analogue of Eq. 3.

As noted earlier, many models of dispersal, particularly for coral reefs, assume that the onset and duration of competence is the same for all larvae in a cohort, and that mortality rates are constant over time. To determine how violation of these assumptions changes dispersal potential, we compared Eq. 12 with two alternative models that make these more conventional assumptions about competence and survival. To model constant mortality, we simply apply Eq. 3, using the exponential survival model (Eq. 8) for $s(t)$, but otherwise use the same model structure. To model a fixed 
competence period, we use the fact that, in our full model, the mean time to acquire competence is $a^{-1}$ (or $t_{\mathrm{c}}$ $+a^{-1}$ if there is a time lag) and the mean time to lose competence is $a^{-1}+b^{-1}$ (or $t_{\mathrm{c}}+a^{-1}+b^{-1}$ ). The fixed competence model assumes that all larvae acquire and lose competence at these times, yielding

$$
L_{2}(x)=\int_{t=(1 / a)}^{(1 / a)+(1 / b)} D(x, t) s(t) d t .
$$

\section{EMPIRICAL Methods}

To characterize competence dynamics, we used larvae from cohorts of five common broadcast-spawning species: Acropora gemmifera, A. humilis, A. millepora, A. valida, Goniastrea retiformis, and Platygyra daedalea (see Plate 1). Larvae were cultured following the methods of Babcock et al. (2003). Once larvae were motile (two to three days post fertilization) water was changed every three days until larvae were two weeks old, after which water was changed weekly. Water was changed by concentrating larvae in a plankton mesh sieve and placing them in fresh filtered sea water (FSW). Larval cohorts were maintained in $3.5-\mathrm{L}$ polystyrene containers containing $0.2 \mu \mathrm{m}$ FSW to deprive them of the cues required to induce settlement. Initial stocking densities were approximately 1000 larvae/L (Morse et al. 1996). Periodically after spawning, between 5 and 100 of the surviving larvae were pipetted from the cultures and introduced into replicate $500-\mathrm{mL}$ plastic buckets containing a single conditioned clay tile. Tiles were conditioned by placing them in a horizontal position on the reef crest at $2 \mathrm{~m}$ at Orpheus Island Research Station for two months. This period is sufficient for tiles to develop a fouling community with sufficient crustose coralline algae to induce larval settlement (Babcock 1992, Baird et al. 2003). These larvae were left for $72 \mathrm{~h}$, after which the tiles were removed and the number of larvae successfully completing metamorphosis recorded. Metamorphosis was defined as the deposition of a basal disc, which is visible through the tissue of the polyp. Experiments were performed in the laboratory under controlled temperature $\left(26-28^{\circ} \mathrm{C}\right)$ under cool fluorescent lights with a 12:12 h light: dark photoperiod. Sampling intervals were shorter early in larval life, to better characterize the onset and peak of competence, then the sampling interval was reduced to ensure availability of larvae for competence assays until competence approached zero. The intervals also varied among species because initial cohort size, and larval mortality, varied among species.

Larval survivorship was determined periodically from density estimates in replicate $50 \mathrm{~mL}$ subsamples following agitation of the larval cultures. This protocol was followed until the total number of larvae in cultures dropped below approximately 200, at which point all larvae remaining in culture were counted. As with competence assays, sampling intervals were shorter early in larval life to better capture any early, rapid changes in survivorship.

Competence and survival models were fitted to these empirical data using maximum-likelihood methods. For competence, we estimated $a, b$, and (for the time delay model) $t_{\mathrm{c}}$ by fitting Eqs. 5 and 7 with a binomial error structure. For our survival experiments, total larval densities were estimated by subsampling. Because standard survival analyses assume complete censuses of the study population, such methods were not appropriate. Instead, we modeled counts as following a Poisson distribution with mean

$$
\omega_{x}= \begin{cases}f_{0} L_{0} & x=0 \\ f_{x}\left[L_{x-1}-R_{x-1}\right] \frac{s\left(t_{x}\right)}{s\left(t_{x-1}\right)} & x>0\end{cases}
$$

where $f_{x}$ is the (known) fraction of the total volume of water that is sampled at census time $x$, and $R_{x-1}$ is the (known) number of larvae removed just after census time $x-1$ for settlement competence experiments. The expression $s($.$) is the appropriate survival function (Eqs.$ 8,9 , or 10 ), with estimated parameters $\lambda$ and (where appropriate) $v$ and $\sigma ; L_{0}$ is the estimated initial larval population size; and $L_{x-1}$ is the estimated larval abundance at census $x-1$, obtained from

$$
L_{x-1}=\frac{\omega_{x-1}}{f_{x-1}} .
$$

For both competence and survival analyses, we used likelihood ratio tests for model selection in both cases, and obtained confidence limits from profile likelihood intervals.

\section{RESUlts}

\section{Larval competence}

Likelihood ratio tests indicated support for a timedelay of approximately three days for three of the species (A. millepora, A. gemmifera, and $P$. daedalea), before competence began to be acquired (Fig. 1, Table 1). For the other two species ( $A$. valida and $G$. retiformis), competence was already close to its peak value by the first competence assay (Fig. 1B,D); therefore, the time delay was not statistically distinguishable from zero, and the model without a time delay was favored (Table 1). Those species exhibiting a significant delay did not necessarily have the most rapid acquisition of competence: A. millepora, with a three-day time delay, and $A$. valida, with no time delay, had the lowest rates of acquisition of competence $(0.18$ $\mathrm{d}^{-1}$ and $0.22 \mathrm{~d}^{-1}$, respectively), while $A$. gemmifera (with a time delay) and Goniastrea retiformis (with no delay) had much higher rates of acquisition of competence.

The per capita rate of loss of competence, $b$, varied approximately five-fold among species, but was always 

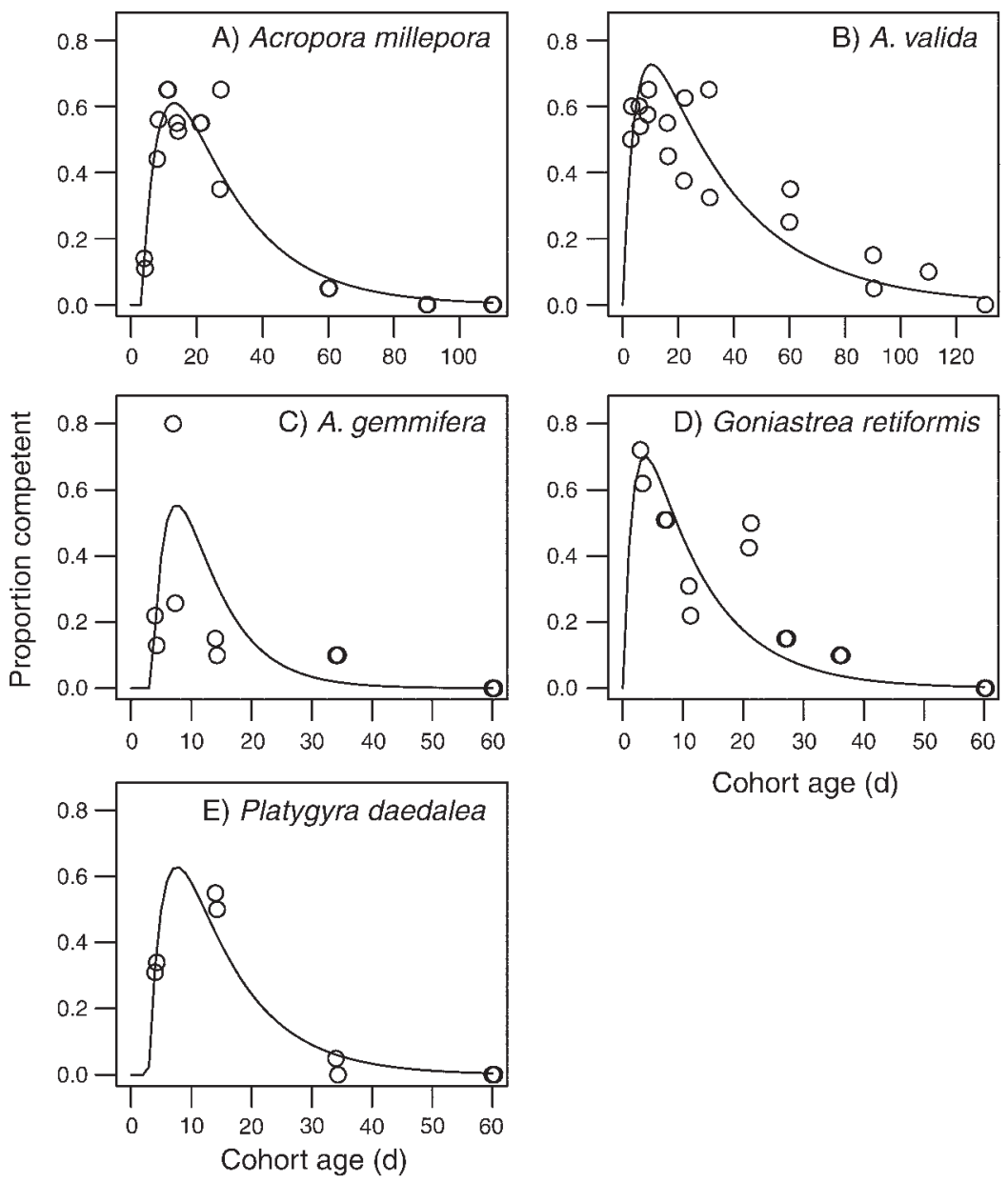

FIG. 1. Observed values (circles) and best-fit models (solid lines) for the proportion of the surviving larval cohort that are competent to settle as a function of time. Fitted model values were generated using either the model without (Eq. 5) or with (Eq. 7) a time delay, depending on which model was favored by model selection (Table 1).

substantially lower than the rate of acquisition of competence (Table 1). Thus, competence in all species peaked relatively quickly $(4-13 \mathrm{~d})$, then underwent a prolonged, approximately exponential decline. Compe- tence was lost most rapidly in $A$. gemmifera, followed by $P$. daedalea, $G$. retiformis, A. millepora and $A$. valida. Note that the reciprocal of the competence loss rate, $b^{-1}$, is the mean duration of the competence period. These

TABLE 1. Maximum-likelihood estimates of rates of acquisition ( $(\hat{a})$ and loss $(\hat{b})$ of competence, and (where appropriate) time lag $\left(\hat{t}_{\mathrm{c}}\right)$.

\begin{tabular}{lccccc}
\hline \hline & & & & \multicolumn{2}{c}{ Likelihood ratio test } \\
\cline { 3 - 6 } \multicolumn{1}{c}{ Species } & \multicolumn{1}{c}{$\hat{a}$} & $\hat{b}$ & $\hat{t}_{\mathrm{c}}$ & Statistic & $P$ \\
\hline Acropora millepora & $0.18(0.14-0.24)$ & $0.050(0.040-0.062)$ & $3.239(2.729-3.553)$ & 39 & $<0.0001$ \\
Acropora valida & $0.22(0.19-0.27)$ & $0.031(0.027-0.036)$ & N/A & 0.000 & $>0.999$ \\
Acropora gemmifera & $0.39(0.25-0.65)$ & $0.145(0.107-0.192)$ & $3.471(3.025-3.717)$ & 29 & $<0.0001$ \\
Goniastrea retiformis & $0.58(0.43-0.83 ; 3.08+) \dagger$ & $0.096(0.086-0.107)$ & N/A & 0.000 & $>0.999$ \\
Platygyra daedalea & $0.39(0.15+)$ & $0.099(0.070-0.137)$ & $2.937(0.442-3.999)$ & 4.67 & $<0.05$ \\
\hline
\end{tabular}

Notes: Values in parentheses indicate $95 \%$ profile likelihood intervals. The last column shows the results of a likelihood ratio test of the models with vs. without a time lag; $P<0.05$ indicates that there is statistically significant support for the more complex timelag model. Model selection by $\mathrm{AIC}_{\mathrm{c}}$ yields the same best-fitting models as likelihood ratio tests.

* The "+" symbol indicates that there was no upper bound to the $95 \%$ confidence region; i.e., the upper bound to the rate of acquisition of competence includes instantaneous acquisition at time 0 (for G. retiformis) and $t_{\mathrm{c}}$ (for $P$. daedalea). dquo; $\mathrm{N} / \mathrm{A}$ " indicates that the best-fitting model omitted this parameter.

$\dagger$ For $G$. retiformis, profile likelihood intervals on the competence acquisition rate $a$ indicated two distinct confidence regions, one extending from 0.43 to 0.83 , and another for $a>3$. The log-likelihood function exhibited an upper asymptote with increasing $a$; therefore, a true maximum-likelihood estimate could not be obtained. 

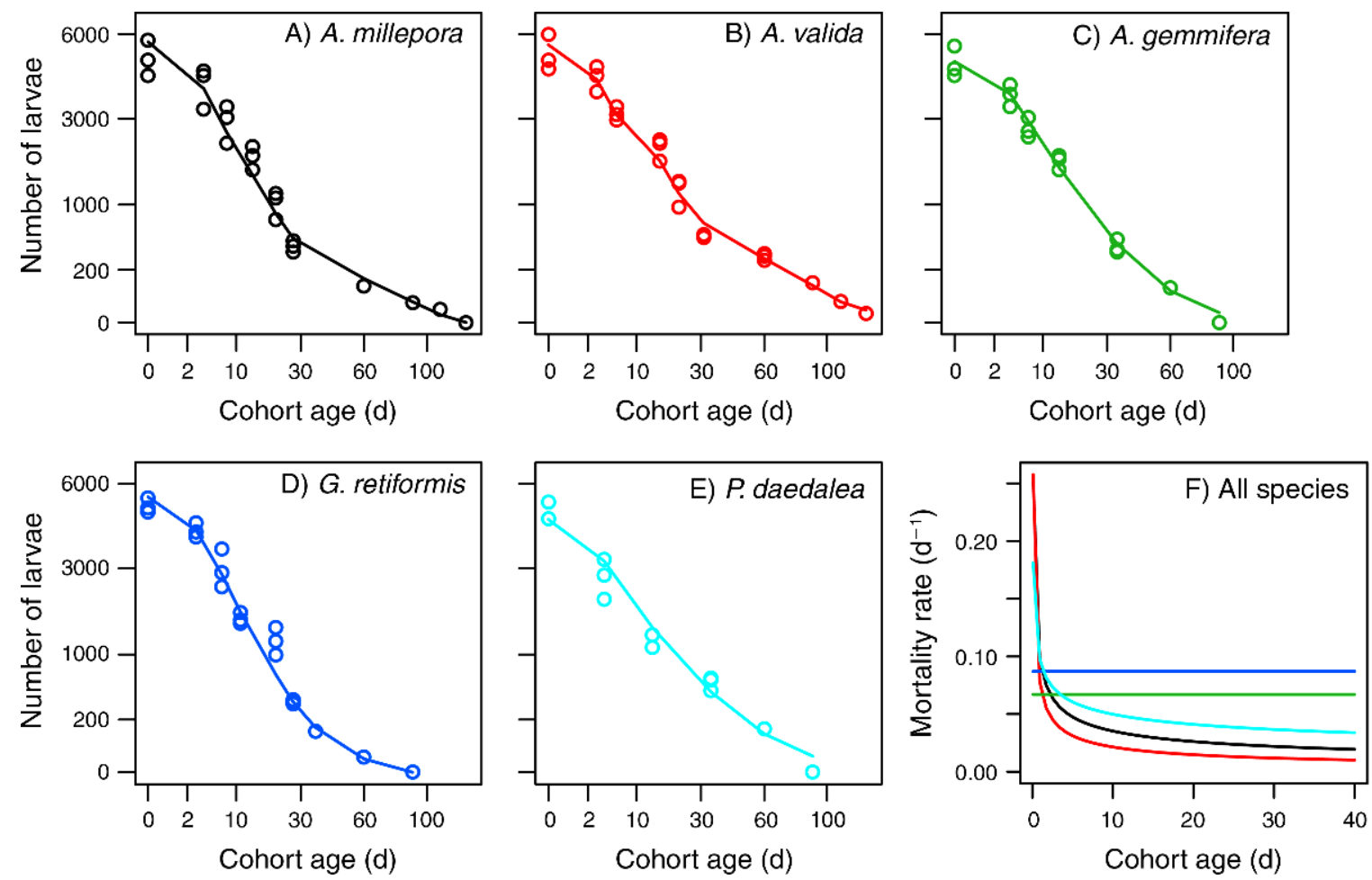

FIG. 2. (A-E) Number of larvae remaining over time in the survival experiments. Circles show the estimated total number of larvae based on the subsample counts. Lines represent survival model predictions (Eq. 15), incorporating the best-fitting survival model as determined by model selection. All axes are plotted on a square-root scale. (F) A comparison of best-fit mortality rate curves for the five study species, based on parameter estimates from the model fits shown in panels A-E.

mean competence periods ranged from 7 to $33 \mathrm{~d}$, and they were identical, in rank order, to the maximum time at which settlement was observed (A. gemmifera, $34 \mathrm{~d} ; P$. daedalea, 34 d; G. retiformis, 36 d; A. millepora, 60 d; A. valida, $110 \mathrm{~d}$ ).

\section{Larval survival}

Survival models provided extremely good fit to the empirical survival data (Fig. 2A-E). To avoid dense clusters of points near axes, and facilitate visual inspection of model fit, axes in Fig. 2 have been square-root transformed. Consequently, changes in the slope of the best-fit line do not correspond to changes in mortality rates. However, parameter estimates and model selection, which do allow a rigorous assessment of time-varying mortality, provide strong evidence for elevated initial mortality in three species, but only weak evidence for elevated mortality late in life (Table 2). Specifically, generalized Weibull parameters were, for all species except $A$. gemmifera, consistent with "bathtubshaped" (decreasing, then increasing) mortality rates $(\sigma$ $>0, v<1)$. However, model selection always rejected this model in favor of one of the simpler ones. For $A$. millepora, $A$. valida, and $P$. daedalea, model selection favored the Weibull model, and, for all species, Weibull parameter estimates indicated high initial mortality that decreased as larvae aged in all cases (i.e., $v<1$; Fig. 2 F).
For A. gemmifera and G. retiformis, model selection favored the exponential model, suggesting that mortality rates in these two species were approximately constant over time (Fig. 2F).

Despite similarities in the shape of the survivorship curves, there were discernable differences in overall survivorship among the five species (Appendix). Specifically, over most of the study period $(t>10 \mathrm{~d}), A$. valida had the highest survival, followed by $A$. millepora, $P$. daedalea, A. gemmifera, and G. retiformis. Except for $G$. retiformis, this is identical to the rank ordering for the mean competence duration, $b^{-1}$, indicating that species with high survival also retain competence longer. In addition, early survivorship was variable among species, with some evidence of a trade-off between high initial and later survivorship: A. valida and A. millepora, for instance, had the lowest survivorship during the initial phase, whereas $A$. gemmifera and $G$. retiformis had the highest.

\section{Dispersal potential}

Integrating survival and competence indicates marked differences in dispersal potential among species (Fig. 3). The proportion of larvae both alive and competent peaked within a week after spawning in $A$. gemmifera, $P$. daedalea, and $G$. retiformis, then dropped concomitantly to about $10 \%$ at $\sim 15-20 \mathrm{~d}$. In contrast, the proportion 
TABLE 2. Parameter estimates and maximized log-likelihoods (MLL) for the survival models, and model comparisons by likelihood ratio test.

\begin{tabular}{|c|c|c|c|c|c|c|c|c|}
\hline \multirow[b]{2}{*}{ Model } & \multirow[b]{2}{*}{$\hat{\lambda}$} & \multirow[b]{2}{*}{$\hat{v}$} & \multirow[b]{2}{*}{$\hat{\sigma}$} & \multirow[b]{2}{*}{ MLL } & \multicolumn{2}{|c|}{ GW vs. Weibull } & \multicolumn{2}{|c|}{ Weibull vs. Exponential } \\
\hline & & & & & Statistic & $P$ & Statistic & $P$ \\
\hline $\begin{array}{l}\text { A. millepora } \\
\text { GW } \\
\text { Weibull } \\
\text { Exponential }\end{array}$ & $\begin{array}{c}0.044 \\
\mathbf{0 . 0 4 3}(\mathbf{0 . 0 3 7 - 0 . 0 4 8 )} \\
0.050\end{array}$ & $\begin{array}{c}0.58 \\
0.57(0.50-0.67)\end{array}$ & -0.009 & $\begin{array}{r}-84.736 \\
-\mathbf{8 4 . 7 3 8} \\
-117.009\end{array}$ & 0.005 & 0.94 & 64.5 & $<0.0001$ \\
\hline $\begin{array}{l}\text { A. valida } \\
\text { GW } \\
\text { Weibull } \\
\text { Exponential }\end{array}$ & $\begin{array}{c}0.022 \\
\mathbf{0 . 0 1 9}(\mathbf{0 . 0 1 2 - 0 . 0 2 3 )} \\
0.36\end{array}$ & $\begin{array}{c}0.48 \\
\mathbf{0 . 4 6}(\mathbf{0 . 4 1 - 0 . 5 9 )}\end{array}$ & -0.108 & $\begin{array}{r}-77.817 \\
-77.858 \\
-107.081\end{array}$ & 0.081 & 0.78 & 58.4 & $<0.0001$ \\
\hline $\begin{array}{l}\text { A. gemmifera } \\
\text { GW } \\
\text { Weibull } \\
\text { Exponential }\end{array}$ & $\begin{array}{c}0.059 \\
0.066 \\
\mathbf{0 . 0 6 7}(\mathbf{0 . 0 6 3 - 0 . 0 7 0 )}\end{array}$ & $\begin{array}{l}1.00 \\
1.08\end{array}$ & 0.189 & $\begin{array}{l}-55.626 \\
-55.931 \\
-\mathbf{5 6 . 3 5 1}\end{array}$ & 0.610 & 0.43 & 0.840 & 0.36 \\
\hline $\begin{array}{l}\text { G. retiformis } \dagger \\
\text { GW } \\
\text { Weibull } \\
\text { Exponential }\end{array}$ & $\begin{array}{c}0.248 \\
0.086 \\
\mathbf{0 . 0 8 7}(\mathbf{0 . 0 8 3 - 0 . 0 9 2})\end{array}$ & $\begin{array}{l}4.48 \\
0.97\end{array}$ & -6.122 & $\begin{array}{l}-73.975 \\
-75.918 \\
-\mathbf{7 5 . 9 7 2}\end{array}$ & 3.89 & $<\mathbf{0 . 0 5}$ & 0.107 & 0.74 \\
\hline $\begin{array}{l}\text { P. daedalea } \\
\text { GW } \\
\text { Weibull } \\
\text { Exponential }\end{array}$ & $\begin{array}{c}0.039 \\
\mathbf{0 . 0 6 0}(\mathbf{0 . 0 5 6 - 0 . 0 6 5 )} \\
0.058\end{array}$ & $\begin{array}{c}0.57 \\
\mathbf{0 . 7 2}(\mathbf{0 . 5 1 - 0 . 9 7 )}\end{array}$ & 0.485 & $\begin{array}{l}-66.516 \\
-67.761 \\
-69.974\end{array}$ & 2.49 & 0.11 & 4.43 & $<0.05$ \\
\hline
\end{tabular}

Notes: GW indicates the generalized Weibull model. A statistically significant result in the model comparisons indicates rejection of the simpler model in favor of the more complex one (all tests are on $1 \mathrm{df}$ ). The best-fit models are indicated in boldface type. For all species, model selection by $\mathrm{AIC}_{\mathrm{c}}$ chooses the same best-fit model as the likelihood ratio tests. Estimated parameters are: $\hat{\lambda}$, the survival rate parameter; $\hat{v}$, a shape parameter of the Weibull and generalized Weibull distributions; and $\hat{\sigma}$, the second shape parameter of the generalized Weibull distribution.

$\dagger$ For G. retiformis, the likelihood ratio test comparing the exponential and Weibull models favors the exponential model, but the test comparing the Weibull and generalized Weibull models marginally favors the generalized Weibull. Therefore, we also compared the generalized Weibull and exponential models directly. This test favors the exponential model (statistic $=3.99, P=0.14$ : note that this test is conducted on two degrees of freedom, not one, because the generalized Weibull has two more parameters than the exponential).

of $A$. valida and A. millepora larvae alive and competent peaked $8-10 \mathrm{~d}$ after spawning and dropped much more gradually (Fig. 3A). When physical transport is incorporated, all species exhibit very leptokurtic dispersal potential (i.e., narrow peaks and thick tails, relative to a Gaussian distribution; Fig. 3B).

Comparison of our best fit models of dispersal potential with the fixed competence and constant survival models indicates that both within-cohort variation in competence dynamics and temporal variation in mortality rates qualitatively change dispersal potential (Fig. 4). Specifically, the fixed competence model underestimates the proportion of the cohort that could settle both very near to and very far from the site of origin (Fig. 4A-E). For A. millepora, A. valida, and, to a lesser extent, $P$. daedalea, the constant survival model also underestimates the proportion of larvae that could settle far from the site of origin, but it overestimates the proportion that could settle very near to the site of origin (Fig. 4F, G, J). Because there was no significant support for time-varying mortality in the other two species, the constant survival model was identical to the best-fit full model for A. gemmifera and G. retiformis (Fig. 4H, I).

\section{DisCUSSION}

Most existing approaches to modeling dispersal assume that all larvae in a cohort acquire competence at the same time, and lose competence at the same time, implying a rectangle-shaped competence pattern. Our models, which are based instead on rates of acquisition and loss of competence within cohorts, provide a better characterization of empirical competence dynamics in scleractinian corals. Specifically, the models capture the fact that peak competence is less than $100 \%$ of the cohort. They also capture the protracted, approximately exponential declines in competence after this peak competence is reached, which imply high within-cohort variation in the duration of competence periods. This variability in competence dynamics increases the potential for both self-recruitment (i.e., settlement at the natal site), and long-distance dispersal. For three of our study species, we also find strong evidence for time-varying larval mortality rates, in violation of the assumption of constant mortality that is commonly made in dispersal models. This implies lower self-recruitment and higher long-distance dispersal than under the conventional constant-mortality assumption. In our study, this latter effect is smaller in magnitude than that produced by 

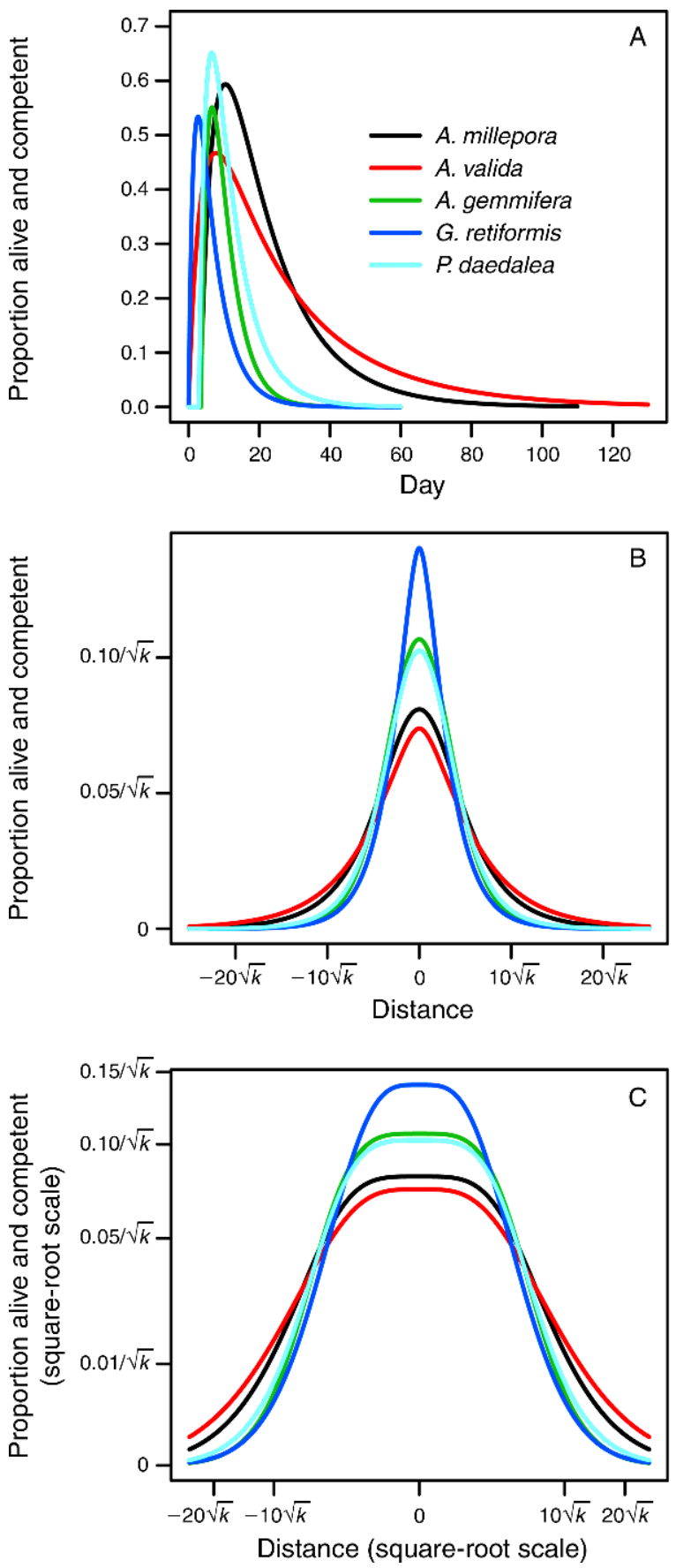

FIG. 3. Predicted dispersal potential as a function of (A) time and (B) distance for the five study species, based on the competence and survival data. In panel (B), the diffusion parameter $k$ influences the scale, but not the shape, of the dispersal kernel, so we have expressed horizontal and vertical axis values as functions of $k$. To facilitate comparison of their shapes, the curves in panel (B) have been normalized to integrate to the same value. Panel (C) shows the same curves as panel (B), but with axes on a square-root scale to facilitate comparison of the tails of the curves.
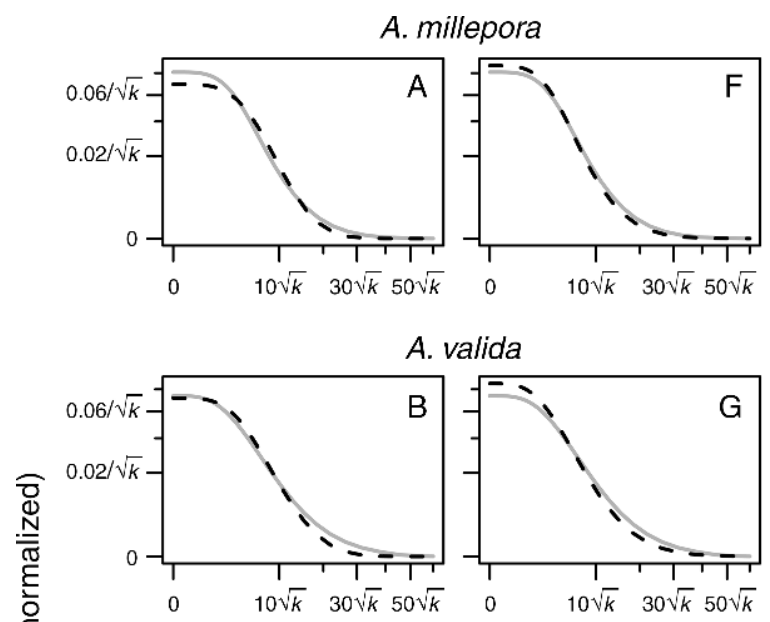

A. gemmifera
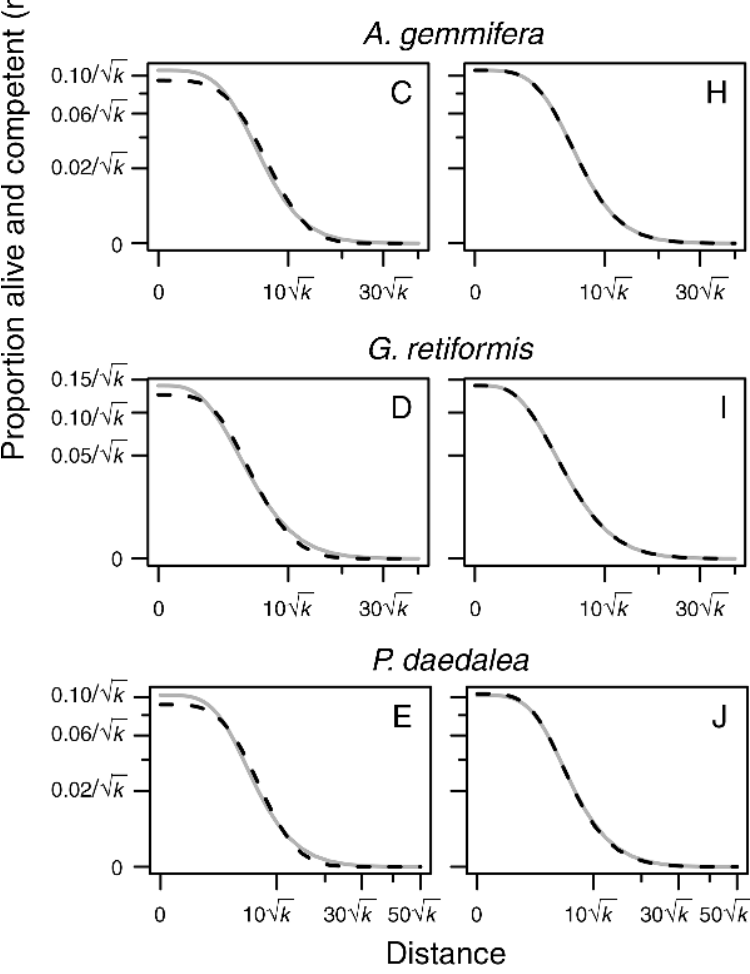

FIG. 4. Comparison of the full calibrated dispersal potential model (solid gray lines) with (A-E) the fixed competence model and $(\mathrm{F}-\mathrm{J})$ the constant survival model (dashed lines). Note that, for (H) A. gemmifera and (I) G. retiformis, the best-fit survival model incorporates a constant mortality rate, so the solid and dashed lines coincide. To facilitate comparison of the curves, horizontal and vertical axes have been plotted on a square-root scale, and curves have been normalized to integrate to the same value. As in Fig. 3B, horizontal and vertical axis values are expressed as functions of the diffusion parameter $k$.

within-cohort variation in competence duration, except for self recruitment of $A$. valida (Fig. 4).

\section{Competence dynamics}

Within-cohort variation in the acquisition and loss of competence makes dispersal potential more leptokurtic than predicted by models that omit such variation (Fig. 
4A-E). This leptokurtic shape means that, if the opportunity presents itself, a higher proportion of coral larvae will self-recruit (i.e., recruit back to the natal reef) than one would predict based on the assumption of fixed times of acquisition and loss of competence. However, if larvae are transported away from their natal reef into the open ocean before competence is acquired, a larger proportion of the cohort also will retain the ability to complete metamorphosis for extended periods.

Our idealized model of physical transport was used to compare dispersal potential for models with and without within-cohort variation in competence. However, physical transport in real reef systems will be less spatially homogeneous than these models imply. Specifically, we assumed a constant diffusion parameter, but, in nature, the extent of diffusive mixing will depend upon the spatial scale of the predominant oceanographic processes (Okubo 1994). In reef systems, for instance, complex local circulation around reefs will influence local retention and self-seeding, whereas dispersal among reefs will be more influenced by mesoscale processes. We expect such characteristics of circulation in reef systems to amplify the effects of within-cohort variation in competence, by increasing the extent of both selfrecruitment, and long-distance dispersal, relative to our simple diffusion model. In particular, hydrodynamic modeling indicates that mean retention times of larvae on reefs can vary from a few hours to more than a week, depending on local wind conditions (Black et al. 1991), a range that is highly consistent with observed retention times of coral "spawning slicks" (visually apparent aggregations of larvae) in reef lagoons (Willis and Oliver 1988, Gilmour et al. 2009). The longer retention times associated with quiescent conditions are more than adequate for a substantial proportion of the cohort to attain competence and settle while still on the natal reef (Fig. 3A). However, when strong surface currents transport larvae off the reef quickly, dispersal will be determined principally by mesoscale processes. In such cases, the thicker tails associated with within-cohort variation in competence will substantially increase the proportion of larvae that may be transported long distances (Fig. 4). Qualitatively similar complexities are likely to hold in other systems. For instance, larvae from coastal intertidal and subtidal habitats may be locally retained by tidal fronts and other nearshore circulation features over short time scales, with mesoscale features dominating when larvae escape these retention zones (Lagos et al. 2008). However, because their competence acquisition times correspond closely with local retention times, variation in competence duration may be a particularly important determinant of dispersal potential in scleractinian corals. This may help to explain why they have relatively high levels of spatial population genetic structure, but also very large ranges, compared with many other marine organisms (Paulay and Meyer 2002, Hellberg 2007).

\section{Survival dynamics}

We found support for elevated mortality rates early, but not late, in larval life. This appears to differ from earlier work showing both phenomena in some of the same species (Graham et al. 2008). However, this discrepancy is probably due to differences in the duration of our experiments, rather than real differences in survival patterns. Specifically, Graham et al. (2008) found increasing mortality rates after about $100 \mathrm{~d}$, whereas, due to the repeated removal of larvae for settlement assays in the present study, only $A$. valida and $A$. millepora larvae were still alive after 100 days, and all cultures had expired by 130 days. Thus, we would have been unlikely to detect increases in mortality rate this late in larval life. Nevertheless, we suspect that the effect of such increases in mortality would have a relatively small effect on dispersal potential, because most of the few remaining larvae still alive at the onset of senescence would have already lost competence. For instance, based on our parameter estimates at 100 days, less than $1 \%$ of larvae of any of our study species would be alive and competent to settle. Moreover, 100 days is more than adequate for extremely long-distance dispersal (e.g., across the 4000 km East Pacific Divide; Richmond 1987).

\section{Robustness}

Tracking of larval cohorts, and repeated sampling for competence and survival, are rarely (if ever) practical under field conditions. For corals, field data for larval competence encompass only the first few days after spawning for a single study (Gilmour et al. 2009), and we know of no field studies of survival. Consequently, our model calibration was based on laboratory data. Nevertheless, it is important to evaluate the robustness of our principal conclusions to differences between field and laboratory conditions. With respect to competence, field conditions differ from those in the laboratory in ways that are likely to increase within-cohort variation in competence, and thus amplify the effects on dispersal potential identified in our analyses. Specifically, in the laboratory, gametes from a small number of colonies give rise to the larval cohorts. Thus, any variation in competence duration that is due either to genetic variation among parents, or to variation in parental condition, would tend to be greater in the field than in culture. Moreover, larvae in culture are retained in homogeneous conditions, whereas diffusion of larvae in the field means that different larvae experience different environmental conditions (e.g., temperature), which can, in turn, influence development rates or energy expenditure. This too would tend to increase the variability in the acquisition and duration of competence, relative to the laboratory, and thereby lead to even greater relative increases in self-recruitment, and long-distance dispersal, compared to our results. Similarly, spawning, even by individual coral colonies, can be spread over multiple days or even lunar cycles (e.g., Bastidas et al. 2005), such that offspring of the same 


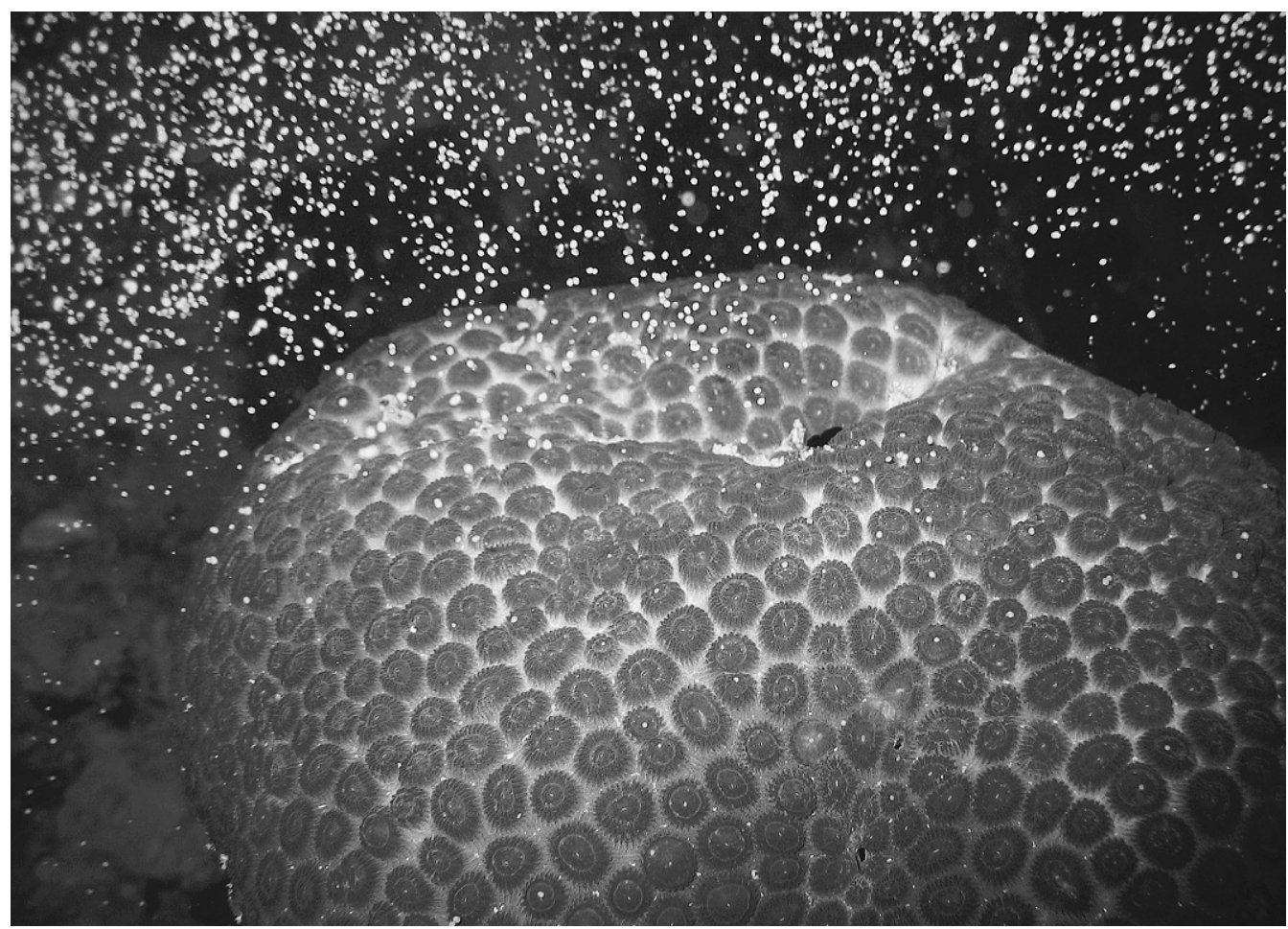

Plate 1. Goniastrea aspera releasing egg sperm bundles two hours after sunset in Palau (western Carolina Islands, in the Philippine Sea) in May 2004. Most corals, including all species used in this study, broadcast-spawn positively bouyant gametes, which then have an obligate planktonic phase of variable duration, depending on the species, before the larvae become competent to settle. Photo credit: A. H. Baird.

parent may be subjected to differing environmental conditions that accelerate or retard the acquisition or loss of settlement competence.

Potential sources of mortality differ in kind and degree under laboratory and field conditions, with implications for overall mortality rates, and for changes in mortality rates over time. Some sources of mortality in the field, such as predation, are not present in the laboratory, and many abiotic stressors, such as UV radiation, and fluctuations in temperature or other physical parameters, are absent or minimized. Indeed, after the first week of our study, mortality rates were relatively low $(\sim 2-8 \%$ per day), whereas values at or above $10 \%$ are commonly reported in the field (Rumrill 1990, Lamare and Barker 1999). It seems unlikely that this affects substantially the qualitative effects of timevarying mortality, or variation in competence duration, found in this study. However, it does suggest that there may be less long-distance dispersal in the field than our mortality estimates imply. Similarly, for lecithotrophic larvae, long-distance dispersal is likely to be associated with depletion of energy reserves, and there is some evidence that this may lead to lower post-settlement survival for at least some marine invertebrates (Pechenik 2006).

With respect to time-varying mortality, larval densities in the laboratory tend to be kept high for logistical reasons, and this can potentially exacerbate the risk of pathogen outbreaks, or mortality of larvae as they become entrained in the lysed tissues of dead larvae. We observed no signs of pathogens in our cultures, suggesting that this was not a major determinant of elevated early mortality. Rather, for our study species, mortality rates had stabilized within 10 days (Fig. 2F). This corresponds approximately to the expected time to peak competence, implicating developmental failure as a likely cause of increased early mortality. We have no reason to expect such problems to be more or less severe under field conditions. However, predation is an additional cause of larval mortality in the field, particularly just after spawning (Pratchett et al. 2001). This could change the magnitude of the initial decline in mortality rates, depending on how increased predator activity trades off against higher densities of larvae immediately after spawning. It seems less likely that predation rates would vary markedly towards the end of the larval phase, when larval densities are much lower. Thus, while we suspect that larval mortality rates in the field are elevated early in larval life, it is less clear whether such effects are larger, or smaller, than those found in this study, and thus whether the predicted increase in self-recruitment arising from this elevated mortality would be larger, or smaller, than our results indicate (Fig. 4F-J). 


\section{CONCLUSIONS AND ImPLiCATIONS}

Estimates of dispersal potential are critical to understanding metapopulation dynamics, population genetic structure, biodiversity, and biogeography. Such estimates are also needed to improve the design of reserve networks (Almany et al. 2009). For marine systems, our capacity to construct realistic, detailed models of the physical mechanisms that drive larval transport has outpaced our ability to characterize biological influences on dispersal (Cowen and Sponaugle 2009). Although there has been increasing attention to the incorporation of larval swimming behavior and position in the water column, particularly for fishes (Paris and Cowan 2004, Paris et al. 2007), competence and survival dynamics have received much less attention, and tend to be characterized relatively crudely in dispersal models. We have shown here that simple, parsimonious models of competence dynamics can substantially improve our characterization of empirical competence patterns. Such models can be readily calibrated and integrated with hydrodynamically realistic models of physical transport in particular regional contexts, and thereby substantially improve estimates of connectivity in those systems. Our findings in this study indicate that modeling within-cohort variation in competence dynamics will change our estimates of both local self-seeding and long-distance dispersal in marine metapopulations.

\section{ACKNOWLEDGMENTS}

This project was funded by PADI AWARE, the Great Barrier Reef Marine Park Authority, the Australian Coral Reef Society, James Cook University, the Royal Zoological Society of NSW, and the Australian Research Council. We thank the staff at Orpheus Island Research Station for their assistance and support. M. Hisano and E. Graham provided assistance with statistical programming and manuscript preparation. Comments from L. Bay, J. Gilmour, V. Harriott, A. Heyward, T. Hughes, M. Kospartov, M. Pratchett, S. Purcell, L. Smith, G. Sneddon, B. Willis, J. Wolstenholme, and two anonymous reviewers greatly improved the manuscript.

\section{Literature Cited}

Almany, G. R., S. R. Connolly, D. D. Heath, J. D. Hogan, G. P. Jones, L. J. McCook, M. Mills, R. L. Pressey, and D. H. Williamson. 2009. Connectivity, biodiversity conservation and the design of marine reserve networks for coral reefs. Coral Reefs 28:339-351.

Babcock, R. C. 1992. Workshop on coral and fish recruitment. Boliano Marine Laboratory, Marine Science Institute, University of the Philippines, Manila, Philippines.

Babcock, R. C., A. H. Baird, S. Piromvaragorn, D. P. Thomson, and B. L. Willis. 2003. Identification of scleractinian coral recruits from Indo-Pacific reefs. Zoological Studies 42:211-226.

Baird, A. H. 2001. The ecology of coral larvae: settlement patterns, habitat selection and the length of the larval phase. Dissertation. James Cook University, Townsville, Australia.

Baird, A. H., R. C. Babcock, and C. P. Mundy. 2003. Habitat selection by larvae influences the depth distribution of six common coral species. Marine Ecology Progress Series 252: 289-293.

Baird, A. H., J. R. Guest, and B. L. Willis. 2009. Systematic and biogeographical patterns in the reproductive biology of scleractinian corals. Annual Review of Ecology and Systematics 40:531-571.

Bastidas, C., A. Croquer, A. L. Zubillaga, R. Ramos, V. Kortnik, C. Weinberger, and L. M. Marquez. 2005. Coral mass- and split-spawning at a coastal and an offshore Venezuelan reefs, southern Caribbean. Hydrobiologia 541: 101-106.

Ben-David-Zaslow, R., and Y. Benayahu. 1998. Competence and longevity in planulae of several species of soft corals. Marine Ecology Progress Series 163:235-243.

Bjornstad, O. N., R. A. Ims, and X. Lambin. 1999. Spatial population dynamics: analyzing patterns and processes of population synchrony. Trends in Ecology and Evolution 14: 427-432.

Black, K., P. Moran, and L. Hammond. 1991. Numerical models show coral reefs can be self-seeding. Marine Ecology Progress Series 74:1-11.

Bohonak, A. J. 1999. Dispersal, gene flow, and population structure. Quarterly Review of Biology 74:21-45.

Chia, F. S., J. Bucklandnicks, and C. M. Young. 1984. Locomotion of marine invertebrate larvae: a review. Canadian Journal of Zoology 62:1205-1222.

Compton, S. G. A. 2002. Sailing with the wind: dispersal by small flying insects. Pages 113-133 in J. M. Bullock, R. E. Kenward, and R. S. Hails, editors. Dispersal ecology. Cambridge University Press, Cambridge, UK.

Cowen, R. K., C. B. Paris, and A. Srinivasan. 2006. Scaling of connectivity in marine populations. Science $311: 522-527$.

Cowen, R. K., and S. Sponaugle. 2009. Larval dispersal and marine population connectivity. Annual Review of Marine Science 1:443-466.

Gallego, A., E. W. North, and P. Petitgas. 2007. Introduction: status and future of modelling physical-biological interactions during the early life of fishes. Marine Ecology Progress Series 347:122-126.

Gilmour, J. P., L. D. Smith, and R. M. Brinkman. 2009. Biannual spawning, rapid larval development, and evidence of self-seeding for scleractinian corals at an isolated system of reefs. Marine Biology 156:1297-1309.

Graham, E. M., A. H. Baird, and S. R. Connolly. 2008. Survival dynamics of scleractinian coral larvae and implications for dispersal. Coral Reefs 27:529-539.

Harrison, P. I., and C. C. Wallace. 1990. Reproduction, dispersal, and recruitment of scleractinian corals. Pages 133-207 in Z. Dubinsky, editor. Coral reefs. Ecosystems of the world. Volume 25. Elsevier, Amsterdam, The Netherlands.

Hellberg, M. E. 2007. Footprints on water: the genetic wake of dispersal among reefs. Coral Reefs 26:463-473.

Hughes, T. P., A. H. Baird, E. A. Dinsdale, N. A. Moltschaniwskyj, M. S. Pratchett, J. E. Tanner, and B. L. Willis. 2000. Supply-side ecology works both ways: the link between benthic adults, fecundity, and larval recruits. Ecology 81:2241-2249.

Hughes, T. P., D. R. Bellwood, and S. R. Connolly. 2002. Biodiversity hotspots, centres of endemicity, and the conservation of coral reefs. Ecology Letters 5:775-784.

Jokiel, P. L. 1990. Transport of reef corals into the Great Barrier Reef. Nature 347:665-667.

Kinlan, B. P., and S. D. Gaines. 2003. Propagule dispersal in marine and terrestrial environments: a community perspective. Ecology 84:2007-2020.

Lagos, N. A., J. C. Castilla, and B. R. Broitman. 2008. Spatial environmental correlates of intertidal recruitment: a test using barnacles in northern Chile. Ecological Monographs 78:245-261.

Lamare, M. D., and M. F. Barker. 1999. In situ estimates of larval development and mortality in the New Zealand sea urchin Evechinus chloroticus (Echinodermata: Echinoidea). Marine Ecology Progress Series 180:197-211. 
Largier, J. L. 2003. Considerations in estimating larval dispersal distances from oceanographic data. Ecological Applications 13:71-89.

Lester, S. E., B. I. Ruttenberg, S. D. Gaines, and B. P. Kinlan. 2007. The relationship between dispersal ability and geographic range size. Ecology Letters 10:745-758.

Miller, K., and C. Mundy. 2003. Rapid settlement in broadcast spawning corals: implications for larval dispersal. Coral Reefs 22:99-106.

Morse, A. N. C., K. Iwao, M. Baba, K. Shimoike, T. Hayashibara, and M. Omori. 1996. An ancient chemosensory mechanism brings new life to coral reefs. Biological Bulletin 191:149-154.

Mouquet, N., and M. Loreau. 2003. Community patterns in source-sink metacommunities. American Naturalist 162: 544-557.

Mudholkar, G. S., D. K. Srivastava, and G. D. Kollia. 1996. A generalization of the Weibull distribution with application to the analysis of survival. Journal of the American Statistical Association 91:1575-1583.

Nathan, R., and H. C. Muller-Landau. 2000. Spatial patterns of seed dispersal, their determinants and consequences for recruitment. Trends in Ecology and Evolution 15:278-285.

Nozawa, Y., and P. L. Harrison. 2008. Temporal patterns of larval settlement and survivorship of two broadcast-spawning acroporid corals. Marine Biology 155:347-351.

Okubo, A. 1994. The role of diffusion and related physical processes in dispersal and recruitment of marine populations. Pages 5-32 in P. W. Sammarco and M. L. Heron, editors. The bio-physics of marine larval dispersal. American Geophysical Union, Washington, D.C., USA.

Palumbi, S. R. 1994. Genetic-divergence, reproductive isolation, and marine speciation. Annual Review of Ecology and Systematics 25:547-572.

Paris, C. B., L. M. Cherubin, and R. K. Cowen. 2007. Surfing, spinning, or diving from reef to reef: effects on population connectivity. Marine Ecology Progress Series 347:285-300.

Paris, C. B., and R. K. Cowan. 2004. Direct evidence of a biophysical retention mechanism for coral reef fish larvae. Limnology and Oceanography 49:1964-1979.

Paruntu, C. P., K. Hidaka, and M. Hidaka. 2000. Developmental changes in cnida composition of the coral Pocillopora damicornis. Galaxea 2:23-38.

Paulay, G., and C. Meyer. 2002. Diversification in the tropical Pacific: comparisons between marine and terrestrial systems and the importance of founder speciation. Integrative and Comparative Biology 42:922-934.

Pechenik, J. A. 2006. Larval experience and latent effects: metamorphosis is not a new beginning. Integrative and Comparative Biology 46:323-333.

Pratchett, M. S., N. Gust, G. Goby, and S. O. Klanten. 2001. Consumption of coral propagules represents a significant trophic link between corals and reef fish. Coral Reefs 20:1317.

Richmond, R. H. 1987. Energetics, competence, and longdistance dispersal of planula larvae of the coral Pocillopora damicornis. Marine Biology 93:527-533.

Rumrill, S. S. 1990. Natural mortality of marine invertebrate larvae. Ophelia 32:163-198.

Siegel, D. A., S. Mitarai, C. J. Costello, S. D. Gaines, B. E. Kendall, R. R. Warner, and K. B. Winters. 2008. The stochastic nature of larval connectivity among nearshore marine populations. Proceedings of the National Academy of Sciences USA 105:8974-8979.

Sponaugle, S., R. K. Cowen, A. Shanks, S. G. Morgan, J. M. Leis, J. S. Pineda, G. W. Boehlert, M. J. Kingsford, K. C. Lindeman, C. Grimes, and J. L. Munro. 2002. Predicting selfrecruitment in marine populations: Biophysical correlates and mechanisms. Bulletin of Marine Science 70:341-375.

Strathmann, R. R. 1974. The spread of sibling larvae of sedentary marine invertebrates. American Naturalist 108:29 44.

Strathmann, R. R., T. P. Hughes, A. M. Kuris, K. C. Lindeman, S. G. Morgan, J. M. Pandolfi, and R. R. Warner. 2002. Evolution of local recruitment and its consequences for marine populations. Bulletin of Marine Science 70:377-396.

Tilman, D., C. L. Lehman, and C. Yin. 1997. Habitat destruction, dispersal, and deterministic extinction in competitive communities. American Naturalist 149:407-435.

van Oppen, M. J. H., and R. D. Gates. 2006. Conservation genetics and the resilience of reef-building corals. Molecular Ecology 15:3863-3883.

Vikebo, F., C. Jorgensen, T. Kristiansen, and O. Fiksen. 2007. Drift, growth, and survival of larval Northeast Arctic cod with simple rules of behaviour. Marine Ecology Progress Series 347:207-219.

Willis, B. L., and J. K. Oliver. 1988. Inter-reef dispersal of coral larvae following the annual mass spawning on the Great Barrier Reef. Pages 853-859 in Proceedings of the 6th International Coral Reef Symposium, Townsville, Australia.

\section{APPENDIX}

Comparison of best-fit survival curves for the five study species (Ecological Archives E091-251-A1). 